\title{
KEEPING PEOPLE SMILING
}

TANDEX has added international standard ISO and PHD (passage hole diameter) numbers to the packaging of its FLEXI interdental brushes.

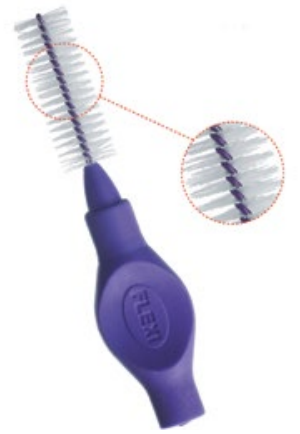

The ISO defines what PHD interval the brush can be squeezed into without deformation. PHD is based on the number of filaments per $\mathrm{cm}$, nylon thickness per $\mathrm{mm}$ and how hard the brush is twined. Dental practitioners can help patients find the right-size brush for them, meaning elevated daily cleaning.

This means improved oral health, general health and wellbeing too!

TANDEX wants to keep people smiling - find out more about all its products, for daily at-home preventive care, today.

For more information on Tandex's range of products, visit www.tandex.dk.

\section{NEW CLINICALLY PROVEN NON-INVASIVE TREATMENT FOR SNORING}

Innovators in challenging conventional snoring and sleep apnoea treatments, Signifier Medical Technologies has created the first and only daytime therapy that tackles the root cause of sleep disordered breathing. The Snoozeal device has demonstrated significant clinical reductions in snoring and mild obstructive sleep apnoea (OSA) of approximately $50 \%$ on objective assessments. Furthermore $80 \%$ of bed partners perceived improvement in snoring (average $40 \%$ improvement), and quality of sleep for both them, and their partner in recent medical trials in over 100 patients. ${ }^{1,2,3}$

Snoozeal is a non-invasive daytime training therapy for the tongue which delivers small contractions that improves muscle function and reduces the physiological collapse of the tongue during sleep. The device is a one size fits all and effective when used for 20 minutes a day for six weeks, with sustained improvement with minimal weekly maintenance.

There is a vast unmet therapeutic need in snoring and mild sleep apnoea, with up to nine million adults in the UK. ${ }^{4}$ Patients often find conventional night-time solutions ineffective or difficult to tolerate. With the increasing public and digital awareness of sleep and sleep devices, it is likely that more patients will be coming to their dentist with sleep related issues. Snoozeal gives dentists and patients an effective solution that is easy and convenient to use.

Visit www.snoozeal.com for more information or to sign up for a free webinar.

\section{References}

1. Wessolleck E, Bernd E, Dockter S, Lang S, Sama A, Stuck B A. Intraoral electrical muscle stimulation in the treatment of snoring. Somnologie (Berl) 2018; 22(Suppl 2): 47-52.

2. Sama A et al. Daytime Intraoral Neurostimulation with Snoozeal ${ }^{\circ}$ for treatment of Snoring and Mild Sleep Apnea. CHEST Annual Meeting Notes, 2018.

3. Prospective cohort study of 50 patients with snoring or mild OSA (ApneaHypopnea Index $(\mathrm{AHI})<15)$ with 46 completed the trial. Objective snoring and respiratory parameters were recorded with two consecutive WatchPat night sleep studies before and after the use of the device. An intra-oral tongue stimulator $\left(\right.$ Snoozeal ${ }^{\circ}$ ) device was used for 20 minutess, once a day for 6-week period.

4. Benjafield A V, Ayas N T, Eastwood P R et al. Estimation of the global prevalence and burden of obstructive sleep apnoea: a literature-based analysis. Lancet Respir Med 2019; 7: 687-698.

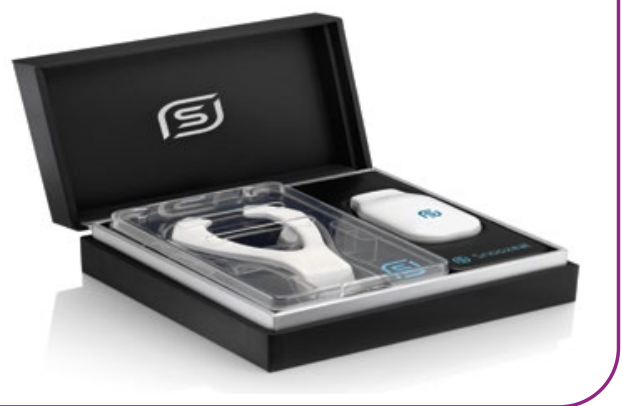

\section{ANTIBODY TESTING FOR YOUR PATIENTS}

Testing has barely got a mention in any of the guidance produced by recognised bodies. Why? Possibly because the UK government has had a poor record with testing to date. Swab tests for COVID-19 which are 'have you got it now?' tests are held up as the standard but are actually only between $50-70 \%$ accurate. They require laboratories and take several days to turn around results. Point of care antigen testing outside of hospitals does not seem to be an option. Good antibody tests, such as Spring Health's, can offer close to $100 \%$ accuracy and if they are highly specific, will not give you false positives. Tests like this can offer a point of care solution.

So what can these tests tell you? If a patient has had symptoms of Coronavirus and has a positive antibody test using an accurate test such as Spring Health's, you can assume with some degree of comfort that they have recovered from COVID-19. It's very unlikely and likely impossible that they will pick this version of Coronavirus up again and pass it on. By screening patients, we can see how much Coronavirus has been passed around our dental community, and how prevalent the disease is. Screening can keep us alert to how much of a problem it continues to be within our own population of patients. Remember some parts of the country and some ethnicities seem to have been worse hit than others. Testing staff regularly and after illness is also important in their new COVID-19 workplace. As the risk level reduces, why not use these tests to stratify patients into risk categories: low risk COVID-19 antibody positive patients, requiring modified PPE (a visor, surgical mask and gloves) and COVID antibody negative patients requiring more protection.

For more information, visit www. salubritas.co.uk or email guidance@ salubritas.co.uk. Tests cost $£ 18$ inc VAT and come in boxes of ten. 